\title{
BMJ Open Delivering dementia care differently- evaluating the differences and similarities between a specialist medical and mental health unit and standard acute care wards: a qualitative study of family carers' perceptions of quality of care
}

\author{
Karen Spencer, ${ }^{1}$ Pippa Foster, ${ }^{1}$ Kathy H Whittamore, ${ }^{2}$ Sarah E Goldberg, ${ }^{1}$ \\ Rowan $\mathrm{H} \mathrm{Harwood}{ }^{1,2}$
}

To cite: Spencer K, Foster $\mathrm{P}$, Whittamore KH, et al. Delivering dementia care differently-evaluating the differences and similarities between a specialist medical and mental health unit and standard acute care wards: a qualitative study of family carers' perceptions of quality of care. BMJ Open 2013:3:e004198

doi:10.1136/bmjopen-2013004198

- Prepublication history and additional material for this paper is available online. To view these files please visit the journal online (http://dx.doi.org/10.1136/ bmjopen-2013-004198).

Received 8 October 2013 Revised 18 November 2013 Accepted 20 November 2013

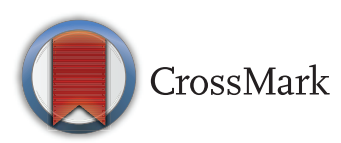

For numbered affiliations see end of article.

Correspondence to Professor Rowan Harwood; rowan.harwood@nuh.nhs.uk

\section{ABSTRACT}

Objectives: To examine in depth carers' views and experiences of the delivery of patient care for people with dementia or delirium in an acute general hospital, in order to evaluate a specialist Medical and Mental Health Unit (MMHU) compared with standard hospital wards. This qualitative study complemented the quantitative findings of a randomised controlled trial.

Design: Qualitative semistructured interviews were conducted with carers of patients with cognitive impairment admitted to hospital over a 4-month period.

Setting: A specialist MMHU was developed in an English National Health Service acute hospital aiming to deliver the best-practice care. Specialist mental health staff were integrated with the ward team. All staff received enhanced training in dementia, delirium and personcentred care. A programme of purposeful therapeutic and leisure activities was introduced. The ward environment was optimised to improve patient orientation and independence. A proactive and inclusive approach to family carers was encouraged.

Participants: 40 carers who had been recruited to a randomised controlled trial comparing the MMHU with standard wards.

Results: The main themes identified related closely to family carers' met or unmet expectations and included activities and boredom, staff knowledge, dignity and fundamental care, the ward environment and communication between staff and carers. Carers from MMHU were aware of, and appreciated, improvements relating to activities, the ward environment and staff knowledge and skill in the appropriate management of dementia and delirium. However, communication and engagement of family carers were still perceived as insufficient.

Conclusions: Our data demonstrate the extent to which the MMHU succeeded in its goal of providing the bestpractice care and improving carer experience, and where
Strengths and limitations of this study

- This study was undertaken alongside a randomised controlled trial, so patients and carers were to an extent matched for social and clinical characteristics. Wide-ranging semistructured interviews permitted exploration of experiences, and uncovered areas of concern that might not have been anticipated in advance.

- Data are limited by coming from a single hospital. Studying the experience of patients with cognitive impairment is difficult because of memory, language and other cognitive problems. Family carers represent a suitable proxy, but have a partial view of the care delivered to a patient.

- Interviews were conducted some weeks after hospitalisation, which might influence perceptions and interpretations, and which aspects of the story were related to the interviewer. Family carers were sometimes interviewed with the patient participant present, which might limit what was said openly. The analysis of qualitative data is open to different interpretations and the possibility of preconception.

deficiencies remained. Neither setting was perceived as neither wholly good nor wholly bad; however, greater satisfaction (and less dissatisfaction) with care was experienced by carers from MMHU compared with standard care wards.

\section{INTRODUCTION}

The prevalence of dementia is increasing worldwide. ${ }^{1}$ One in three acute hospital admissions is of a confused older person. ${ }^{2}$ In recent 
years, various reports have called for improvements in care for people with dementia admitted to hospital. ${ }^{3-7}$ The Alzheimer's Society ${ }^{8}$ identified key areas of dissatisfaction for carers relating to a lack of person-centred care (PCC), nurses not recognising or understanding dementia, a lack of dignity and respect, patients not being helped to eat and drink, a lack of opportunity for social interaction and not enough carer collaboration in decision-making. The qualitative research exploring carers' perceptions of acute hospital care for people with dementia suggest that their experiences are variable. One study concluded that perceptions of poor care were linked to expectations and relationships with staff. ${ }^{9}$ Staff report lacking skills and confidence in caring for confused older people. ${ }^{8}{ }^{10}$ Little previous research works have evaluated the attempts to improve the quality of care for confused older people in acute hospitals.

A specialist Medical and Mental Health Unit (MMHU) was developed on an acute geriatric medical ward aiming to provide the best-practice care for patients with delirium and dementia following admission to a general hospital for acute medical care. ${ }^{11}$ The intervention ward enhanced five aspects of care. Additional specialist staff were employed (mental health nurses, mental health specialist occupational, physiotherapist and speech and language therapists, and a psychiatrist) alongside acute hospital staff. Staff received enhanced training in dementia, delirium and PCC following the Bradford Dementia Group approach ${ }^{12} 13$ using a combination of didactic and ward-based learning, including coworking with the mental health specialist staff. A programme of purposeful activities matched to retained abilities was introduced (such as dominoes, quizzes, crafts, ball games, music and reminiscence, getting dressed and social eating). ${ }^{14}$ The ward environment was optimised with the aim of improving patient orientation and independence. A proactive and inclusive approach to family carers was encouraged, with more communication, liberal visiting times and the invitation to engage in day-to-day care. The patient personal profile documentation was developed to be completed by family carers. A series of information leaflets were designed and made available to carers.

The MMHU was evaluated in a controlled trial that randomised 600 confused patients over the age of 65 who had been admitted for emergency medical care to the unit or standard care. ${ }^{15}{ }^{16}$ Standard care wards comprised $70 \%$ acute geriatric medical and $30 \%$ general medical wards. This study suggested improved patient experience and family carer satisfaction, but no differences in health status outcomes. This qualitative study contributes to the evaluation by exploring carers' accounts of their experiences of hospital care, which is an outcome in itself and a check on the 'fidelity' of the intervention.

\section{METHODS}

\section{Sampling and data collection}

Recruitment to the qualitative study took place over the final 4 months of the trial. During this time, family carers of patients recruited into the trial were asked whether they would also consent to taking part in an interview study. If they agreed, participants were approached by the qualitative researcher, depending only on researcher availability for interviews, until 20 were recruited from each setting. All those approached agreed to take part. Recruitment had to be completed while the trial was on-going, and we chose 20 per group as likely to be sufficient to achieve saturation, although full analysis of data was only completed after recruitment ceased. Carers gave written consent, and took part in a face-to-face semistructured interview. An interview guide was developed and checked in a pilot interview. Initially, topics were selected from the literature, and subsequent topics were added if they arose during interviews. Topics included: patient admission and settling into the ward, carer relationship with staff; the ward environment; patients' daily routines such as sleeping, meals, hygiene and activities; privacy and dignity; care and medical treatment; discharge planning. Participants were encouraged to discuss what they considered worked well and not so well. A series of prompts was devised to encourage participants to elaborate in more detail when asked a general question. Interviews were conducted in the carer's home and consent was obtained to audio record the interviews. Participants were reassured that privacy, confidentiality and identity would be protected. The interviewer was an experienced medical sociologist, not involved in delivering clinical care.

\section{Data analysis}

Interviews were transcribed verbatim, and Nvivo V.10 software was used to facilitate analysis. Data were analysed thematically using a framework analysis that allowed a systematic process to be followed in the development of knowledge and theory. ${ }^{17}$ Framework analysis is a flexible approach utilised in health service research that allows all data to be collected and then analysed. ${ }^{18}$ The organisation of data within this approach involved a five-stage process: (1) familiarisation, (2) identifying a thematic framework, (3) indexing, (4) charting and (5) mapping and interpretation. ${ }^{17}$ Familiarisation with data involved constant comparison across the data to identify categories and themes. Coding transcripts to identify recurrent statements and expressed feelings formed the basis of the thematic framework (see online supplementary appendix 1). Themes were compared and contrasted between settings via indexing, charting and mapping to provide a detailed understanding and interpretation of the participants' experiences, and if and how the intervention added to the carers' perspectives of quality of care. All authors met on a regular basis to discuss the development of codes, themes, categories and theories about the phenomenon being studied.

\section{RESULTS}

\section{Participants}

Twenty carers were interviewed from the MMHU, whose relationship to patients was: 1 spouse, 13 daughters, 
2 sons, 1 brother and 2 grand-daughters. Twenty carers from standard care were: 6 spouses, 6 daughters, 1 grand-daughter, 5 sons, 1 sister and 1 nephew. There were 7 male and 13 female patients from MMHU, with mean age 87 (range 83-97), and 11 male and 9 female patients from standard care, with mean age 85 (range 69-95).

\section{Findings}

Data saturation of the key themes was achieved by interview 15 (MMHU) and interview 18 (standard care). The themes identified as being important in exploring the differences and similarities between the participants' experiences of quality of care in the two groups were:

1. Activities and boredom;

2. Staff knowledge;

3. Dementia, dignity and fundamental care;

4. Ward environment;

5. Communication between carers and staff;

6. Carer expectations.

\section{Activities and boredom}

Carers from both groups commented on the activities offered and whether they perceived that patients experienced boredom. Relatives from MMHU made more references to patients being engaged in activities compared with standard care; half of these relatives were aware, or appreciative, of patient involvement in activities on the ward:

The activity co-ordinator put on some old tunes in the day room, like Frank Sinatra everyone was having a bit of a giggle actually, because she loves music and that stuff, so that's good. (Daughter of 84-year-old, female, MMHU patient.)

Six carers highlighted that patients were too ill to have engaged with activities, or would not have wanted to be involved in these:

We did see the activity room, and I think, possibly, if he'd been well enough to just sit in there whilst things were going on, he would have enjoyed that. (Daughter of 95-year-old, male, MMHU patient)

Four MMHU carers stated that their relative would not have had any lasting memory of activities, and this meant family members would not have been aware what activities their relatives had been involved with. Others were aware that patients had taken part, and felt that even if they had no recollection of this, the patients had nevertheless enjoyed the activities at the time, which carers considered positive:

We went in and she was having her hair done, that person [activity coordinator] said that mum had won the film quiz the night before, and mum couldn't even remember doing it. What a shame that whenever we ask her, she can't remember having done anything. (Daughter of 87-year-old, female, MMHU patient.)

Five relatives from standard care commented that there was little stimulus for patients and some considered that this left patients bored. Others felt that having activities could have prevented behaviours such as wandering or vocalisation. Some standard care relatives referred to the need for the kinds of activities that were being offered on MMHU. A few relatives from MMHU were aware of activities, but felt dissatisfaction because their relatives had not had a chance to engage in them.

\section{Staff knowledge}

There were noticeable differences between the two groups relating to staff knowledge of dementia and delivery of professional care. Carers of patients with MMHU described staff as being 'well prepared' for dealing with confused patients, displaying patience and compassion. Respondents noted that patients who liked to wander were guided by staff when walking up and down rather than constantly being returned to their bed space, a behaviour observed by carers on standard care wards. A few respondents praised the support of mental health nurses on MMHU in defusing situations, although some family carers were unaware that MMHU had special staffing:

One night Grandma was in quite a strop, she was having a tantrum and a nurse came over, she didn't have to and I thought it was nice that she came over and she was trying to calm her. I thought she gave that little bit extra. (Grand-daughter of 91-year-old, female, MMHU patient.)

Standard care respondents felt that some staff displayed a negative attitude towards confused patients. Participants felt that staff had little understanding and limited training in dementia care, which carers felt resulted in patients being ignored, shouted at or threatened when staff were faced with uncooperative or challenging situations. In some cases, this led to a confrontation between nurses and family carers who reacted to what they perceived as unacceptable staff attitudes towards patients. These carers further highlighted that they had not formally reported for fear of repercussions towards their relatives:

She [health care assistant] kept shouting at him, turn over, turn over I can't get to you. So eventually I opened the curtains and said that man's confused he can't understand you. She [health care assistant] knew I was sitting outside the curtain and it didn't deter her, she was really shouting. (Wife of 69-year-old, male, standard care patient.)

Carers further described how they felt it necessary to offer individualised support and guidance to health professionals in dealing with patients, as they considered staff to be lacking in dementia expertise. Some 
participants felt they needed to provide one-to-one personal care as they perceived staff were unable to fulfil this role due to their inexperience. One carer from standard care commented that staff had inferred that the ward was a 'mixed medical ward that was not equipped to deal with dementia patients' who were considered time-consuming, as staff would have to have 'extra patience with them'. Some carers considered offering one-to-one care as an extension of their 'main carer' role which transferred with them from home to hospital:

If he was on a ward where they understood him better and would be able to wash and dress him without me having to go up there, it would have been different. (Daughter of 83-year-old, male, standard care patient.)

\section{Dementia, dignity and fundamental care}

For many family carers an important aspect of satisfaction involved fundamental elements of personal care such as elimination, washing and dressing, eating and drinking. Participants believed that such personal and intimate care should be delivered sensitively and that patient's dignity should be protected. MMHU carers (14) and standard care (10) participants stated that they witnessed appropriate curtain use that ensured patients received privacy when needed. Negative comments from 4 MMHU carers and 10 standard care participants considered that their loved ones had received less dignified care. Concerns from MMHU participants ranged from patients' dentures not being cleaned by staff, the respondents feeling that it was inappropriate for male nurses to attend the toileting needs of female patients and a patient being discharged 'in her night gown, unwashed and smelling'. The complaints raised by standard care participants related to patients being found by visitors with excrement under their fingernails and used incontinence pads found by patients' bedsides. Instances were mentioned of patients undressing in public bays, toilet doors being left open exposing female patients to passing male patients and visitors and a patient being found in wet bed sheets or not washed. This was considered due to staff inexperience in delivering care to confused patients:

I had to clean her nails because she had excrement all under her fingernails, the nurse said she'd put her on a bed pan and then caught her getting off, trying to clear it up. (Son of 87-year-old, female, standard care patient.)

One standard care patient who had recovered from delirium and who was present during an interview with his family carer commented that he had been embarrassed and had not wanted to eat to avoid emptying his bowels after a nurse had not cleaned him properly during a visit to the toilet.

Carers from MMHU (11) and standard care wards (12) expressed satisfaction with the hospital food and positive comments related to the quality, menu choice and patients' enjoyment of meals. Participants from across the sample further highlighted that if frail older patients displayed a poor appetite, hospital staff offered alternative snacks and sandwiches, which carers appreciated. Five carers of patients on MMHU expressed concern about staff not assisting patients with eating and drinking. Dissatisfaction expressed from 10 standard care participants mainly related to a lack of understanding, help and encouragement with eating and/or drinking for confused older patients:

She [patient] wasn't drinking sufficiently, they [staff] were giving her a cup of tea but she couldn't hold it, she'd spill it all down herself. And they [staff] weren't doing anything to help when it was mealtime, they [staff] put the meals out and they [patients] seemed to be left to it. (Son of 93-year-old, female, standard care patient.)

A small number of carers from MMHU (2) and standard care (4) questioned whether confused older patients were in a position to make menu choices about the food they usually chose and enjoyed. Carers commented that staff had probably decided on behalf of patients and would have appreciated more involvement.

\section{Ward environment}

Carers from MMHU and standard care generally described the wards as 'clean and tidy'. Less positive comments were expressed by three carers of patients on standard care related to décor or minor cleanliness issues. Comments made by relatives from MMHU suggested that carers were appreciative of some of the changes made to the ward environment that involved improving the décor and personalising patients' surroundings. Carers were also positive about the day and activities room but very few were aware of the private sensory room. Some carers also considered the availability of information leaflets to be a helpful educational resource:

There's more pictures, and that's nice and there's an activities board which is quite nice as well and there's a bit more colour. They've [patients] all have theses memory boxes behind the bed. Mum hasn't got anything in there yet, but I've got photos that I could put in there. (Daughter of 84-year-old, female, MMHU patient.)

\section{Communication between carers and staff}

Relatives of patients in MMHU and standard care wards had positive and negative experiences of communication and engagement with ward staff, but in general wanted more regular communication. Carers' perceptions of their relationship with staff closely corresponded with their met or unmet expectations, which were influenced by the level of cognitive impairment and communication difficulties experienced by patients. A similar number of carers of MMHU (12) and standard care patients (11) described positive experiences, including certain staff being informative, helpful, friendly or approachable. 
These positive experiences influenced their perceptions of quality of care. However, experiences with different staff members could vary greatly:

We saw [the consultant], who was excellent, he was informative, he was helpful, he was sympathetic, but there was one nurse that came across as abrasive and therefore you're a bit wary about asking too many questions, but the auxiliary nurse was lovely.... (Son of 87-year-old, female, standard care patient)

Family members who described poor relationships or ineffective communication with staff indicated a greater dissatisfaction with the quality of care they experienced. The main grievance cited by carers concerned the lack of being kept informed, which led some to feel neglected and ignored. This point was particularly emphasised by carers who found it difficult or impossible to get information from the patient themselves:

I mean, if people like my dad are in here because of their age and memory loss really, they [staff] should be talking to the family, shouldn't they? Or somebody should. (Daughter of 87-year-old, male, MMHU patient.)

Some family members believed that staff should voluntarily provide information on patient's care and progress rather than families feeling obligated to initiate interactions. Carers who were reluctant to approach staff described feeling anxious about being left uninformed:

I did have to ask to find out what was going on, and I know the ward was busy and you don't want to interfere with people when they're working sort of, but it's kind of, when you're feeling that anxious, you just want that little bit more reassurance that, yes, somebody will come and speak to you. (Grand-daughter of 85-year-old, male, MMHU patient)

Lack of communication and information sharing between staff and family carers was also evident at discharge on both MMHU (9) and standard care (10). Approximately half of the carers described the discharge experience in a number of negative ways including delayed, rushed and undignified. Carers did appreciate that problems were often beyond the control of ward staff themselves and related to organisational barriers:

Discharge was a bit belated. Largely because she had to stay there [in hospital] until the aftercare package with the intermediate care team could be set up to come and supervise her at home. (Husband of 75-year-old, female, MMHU patient.)

In discussions with carers about whether staff had engaged with them about patients' backgrounds and interests, positive and negative comments were noted from the respondents. Half of MMHU carers (10) commented that they had been approached by staff to complete personal profile documentation about patients' past lives. Many considered that they were a good idea although a couple of family members mentioned that they had completed them later than expected, and one respondent speculated whether staff referred to them or not:

I filled one form in I answered, you know, her interests, what she enjoyed doing, I do think it's a good idea. The girl [nurse] that gave me the form said it was, to help them understand the person, to get to know the lady in the bed. (Daughter of 87-year-old, female, MMHU patient.)

Staff on standard care wards did not routinely complete personal profile documentation with family carers, but the respondents were asked whether they felt staff had got to know any background information about patients. Most (15) family carers from standard care commented that staff had not enquired about patients' personal lives other than medical history (compared with four MMHU participants). A few participants considered that it would be difficult for nurses to get to know the patients due to the short length of stay in an acute setting and lack of continuity in care due to shift length and patterns. Some family carers felt that nurses were too busy to have much interaction with patients and that conversation would focus around general tasks such as giving injections, changing drips and other medical treatments:

They [nurses] had conversations with me...what she'd been up to, but not much conversation about her past or anything like that. (Grand-daughter of 98-year-old, female, standard care patient)

\section{Carer expectations}

Relatives' expectations of the quality of care they presumed patients would receive on the ward (formed prior to patient admission) compared with actual experiences (determined by perceptions formed during and after discharge) influenced their satisfaction with the ward. The five themes identified in this analysis (activities, staff knowledge, fundamental care, ward environment and communication between staff and carers) all related to expectations, which formed a cross-cutting theme. In order to examine the unmet expectations, the participants were asked to make suggestions about what future improvements could be made to the ward. Several family carers MMHU (6) and standard care (7) highlighted aspects of communication and collaboration between staff and carers:

I would like it if they [staff] came and introduced themselves. So if they haven't seen you before, then you're sat by your mother's bed, they should come over and say, well, I'm the ward sister, or I'm the daily nurse who's looking after her, you know, sorting her washing [needs] and things like that. (Son of 93-year-old, female, MMHU patient.) 
Further suggestions included more patient stimulation, carers being present during ward rounds, longer visiting hours, having a named nurse, receiving a daily briefing from staff, a daily diary or check lists for carers and separate bays for more vocal patients. Family carers from MMHU and standard care wards had a variety of unique expectations that closely related to their satisfaction or dissatisfaction with care:

I think it's a lot better for the patient if there's continuity with the same person, because then you do get to know that patient a lot, a lot better. But it probably won't work in a hospital situation where I know it's not going to be a one to one situation. It never will be, because the NHS can't afford it. (Son of 89-year-old, male, standard care patient)

I thought he would have been better with mental nurse looking after him. To understand him better, understand his needs, do you know what I mean? Rather than them saying to me, Well, we've tried to talk to your dad this morning and he's just not having none of it, he's a bit confused....No, he's not confused, he's got dementia, he's ill. You know, he's got vascular dementia. (Daughter of 84-year-old, male, standard care patient)

\section{DISCUSSION}

This qualitative study evaluated an intervention which aimed to improve care for patients with dementia and delirium admitted to an acute hospital. The main themes identified in exploring carer satisfaction related closely to their met or unmet expectations and included activities and boredom; staff knowledge; dignity and fundamental care; the ward environment and communication between staff and carers. Neither setting was perceived as wholly good or wholly bad; however, greater satisfaction (and less dissatisfaction) with care was experienced by carers' from MMHU compared with standard care wards. Meeting carers' expectations is an important factor leading to greater satisfaction with patient care. ${ }^{19}$ Clearly developing awareness of carers' expectations/ unmet expectations should enable nursing staff to understand the carers' perspective and improve communication as well as satisfaction. Carers were aware of improvements relating to activities, the ward environment and staff knowledge and awareness of the appropriate management of dementia and delirium. However, in some cases, communication and engagement of family carers were still perceived as insufficient.

The MMHU development was an ambitious attempt to overcome previously identified problems with acute hospital care by acknowledging and attempting to address a lack of knowledge and skills, a bland and disorientating environment, a lack of therapeutic and diversionary activity and better communication and engagement with family carers. ${ }^{11}$ The underlying philosophy was that of PCC, which has been advocated over the past decade in order to enhance well-being for people with dementia and to avoid distress and associated disturbed behaviours. PPC aims to support emotional and psychological needs by valuing people with dementia and treating them as individuals, by looking at the world from the perspective of the person with dementia and by creating a positive social environment. ${ }^{13} 20$ There are various descriptions of PCC but there is no consensus on its definition or how to apply it during acute illness. ${ }^{21}{ }^{22}$ Biographical information can be used to promote knowledge of the person with dementia such as family, occupation, hobbies, likes and dislikes and can help staff to focus on the person as an individual. ${ }^{23}$ Personalising dementia patients' surroundings has been associated with positive effects on behaviour and mood as well as improved orientation. ${ }^{42} 25$ Meaningful activities such as reminiscence, games and crafts can foster social interaction between staff and patients ${ }^{20} 2627$ while dressing and social eating provide a sense of purpose and dignity and maintain necessary basic daily skills. However, hospitals are busy, fast-moving and noisy, making them a difficult place for people with dementia. ${ }^{7}$ Patients are often ill and dependent ${ }^{228}$ and physical medical care is necessarily prioritised. The necessary medical monitoring and nursing interventions can be misunderstood or seen as threatening. The length of stay is typically short. These factors limit the scope for delivering PCC and family engagement. ${ }^{29}$

The central role of family carers as stakeholders in the care of people with dementia has been emphasised many times. ${ }^{30-32}$ Carers' experiences of the wards were mediated by many factors related to the severity of the patients' illness, duration of stay, past experience of hospitalisation, the length of time spent visiting the ward, their expectations of the care the ward could provide, competing commitments and carer strain. ${ }^{33}$ Family carers wanted an even greater level of communication and engagement than was achieved on MMHU, despite this being an explicit goal of the unit (a finding also reported by $\mathrm{Li} e t a l^{34}$ ). Carers were appreciative when involved in aspects of their relative's care but disillusioned when they felt excluded or neglected. Relationships between staff (especially nurses) and relatives still need reforming with more partnership and collaboration. Few previous studies have reported attempts at improvement, and rigorous evaluation is difficult in this field. ${ }^{35}{ }^{36}$ Collaboration, in terms of shared decision-making and exchange of knowledge and information has been shown to be particularly important for relatives' satisfaction with hospital care of older people. ${ }^{37}$ Organisational factors, such as a task focused culture and workload, the organisations' focus on risk, shift patterns and length, a lack of training, poor supervision and resistance to change and bureaucratic issues, have also been identified as impeding the development of effective nursefamily collaboration. ${ }^{10} 38$

\section{Strengths and limitations}

This study was undertaken alongside a randomised controlled trial, so patients and carers were, to an extent, 
matched for social and clinical characteristics, and illness severity. Wide-ranging semistructured interviews permitted exploration of experiences in depth, and uncovered areas of concern for participants that might not have been anticipated in advance. The data are limited by coming from a single English National Health Service hospital, but the hospital provided sole emergency medical services for its local population, and is likely to be representative. Studying the experience of patients with cognitive impairment is difficult because of memory and language problems and difficulties in perceiving time and abstract concepts. Family carers are often assumed to be a suitable proxy, but have a very partial view of the care delivered to a patient. Indeed, they are often aware of this limitation, and it can be a source of anxiety to them. Interviews were conducted some weeks after hospitalisation, which might influence perceptions and interpretations of experiences, and which aspects of the story were related to the interviewer. Family carers were sometimes interviewed with the patient participant present, which might limit what was said openly. The analysis of qualitative data is open to different interpretations and the possibility of preconception.

\section{CONCLUSION}

Critical reports focusing on the dignity and nutrition standards for older patients have prompted an additional focus on patient and carer satisfaction as well as clinical outcomes and safety. ${ }^{39}$ Our findings support recent initiatives to improve care in hospitals. ${ }^{40}$ Dementia 2012, a national challenge calls for improvements in the general hospital care of people with dementia including a better prepared workforce. ${ }^{41}$ The enhancements of care on the MMHU that included enhanced training in dementia, delirium and PCC helped staff to deliver dementia care differently and more appropriately and this study provides evidence of its effectiveness. However, the amount of communication required by family carers cannot be underestimated. We found the extent of this surprising and beyond what we had planned for. New approaches to engage with family carers are required, including the assessment of expectations and the giving and receiving of information. Meeting this need will require major changes to the way acute wards operate, and the reprioritisation of staff time to enable this activity. Facilitating more hands-on care by family members may provide the quid pro quo to enable it within resource-constrained healthcare systems. Organisational development methodologies should be explored in future attempts to implement such changes, alongside more staff-directed education and training interventions and incorporation in preregistration education. Nurse leaders will play an important role in creating conditions and fostering a culture that enables and rewards the delivery of 'relationshipcentred' care for this population. ${ }^{31}$
Author affiliations

${ }^{1}$ Division of Rehabilitation and Ageing, University of Nottingham, Nottingham, UK

${ }^{2}$ Health Care of Older People, Nottingham University Hospitals NHS Trust, Queen's Medical Centre, Nottingham, UK

Acknowledgements The Medical Crises in Older People study group also included Justine Schneider, Simon Conroy, Anthony Avery, Judi Edmans, Adam Gordon, Bella Robbins, Jane Dyas, Pip Logan, Rachel Elliott, Matt Franklin.

Contributors RHH and KS conceived the study. SEG, PF and KHW managed the trial and recruited the participants. KS undertook the interviews, and coded the data. All the authors discussed the coding, and interpretation. KS drafted the manuscript, which was revised by $\mathrm{RHH}$, and approved by all authors. KS is the guarantor.

Funding This article presents an independent research funded by the National Institute for Health Research (NIHR) under its Programme Grants for Applied Research funding scheme (RP-PG-0407-10147).

Competing interests None.

Patient consent Obtained.

Ethics approval The study was approved by the Nottingham research ethics committee.

Provenance and peer review Not commissioned; externally peer reviewed.

Data sharing statement No additional data are available.

Open Access This is an Open Access article distributed in accordance with the Creative Commons Attribution Non Commercial (CC BY-NC 3.0) license, which permits others to distribute, remix, adapt, build upon this work noncommercially, and license their derivative works on different terms, provided the original work is properly cited and the use is non-commercial. See: http:// creativecommons.org/licenses/by-nc/3.0/

\section{REFERENCES}

1. World Health Organization (WHO). Dementia: a public health priority. 2012. http://www.apps.who.int/iris/bitstream/10665/75263/1/ 9789241564458_eng.pdf

2. Goldberg SE, Whittamore K, Harwood RH, et al. The prevalence of mental health problems amongst older adults admitted as an emergency to a general hospital. Age Ageing 2012;41:80-6.

3. Royal College of Psychiatrists. Who cares wins: improving the outcomes for older people admitted to the general hospital: Guidelines for the development of Liaison Mental Health Services for older people. London, 2005. http://www.rcpsych.ac.uk/pdf/ whocareswins.pdf

4. Royal College of Psychiatrists. Report of the National Audit of Dementia Care in General Hospital. In: Young J, Hood C, Woolley R, Gandesha ASouza R. eds London: Healthcare Quality Improvement Partnership. 2011. http://www.rcpsych.ac.uk/pdf/NATIONAL\% 20REPORT\%20-\%20Full\%20Report\%201201122.pdf

5. Department of Health. Living well with dementia: a national dementia strategy: a national dementia strategy. London: Department of Health, 2009. https://www.gov.uk/government/publications/ living-well-with-dementia-a-national-dementia-strategy

6. Department of Health. Quality outcomes for people with dementia building on the work of the national dementia strategy. London: Department of Health, 2010. https://www.gov.uk/government/publications/ quality-outcomes-for-people-withdementia-building-on-the-work-of-the-national-dementia-strategy

7. Royal College of Nursing. Improving quality of care for people with dementia in general hospitals. London: Royal College of Nursing, 2010. http://nursingstandard.rcnpublishing.co.uk/shared/cms/file. asp? $\mathrm{e}=35 \& \mathrm{i}=172190$

8. Alzheimer's Society. Counting the cost: caring for people with dementia in hospital ward. London: Alzheimer's Society, 2009. http:// www.alzheimers.org.uk/countingthecost

9. Jurgens F, Clissett P, Gladman JRF, et al. Why are family carers of people with dementia dissatisfied with general hospital care? A qualitative study. BMC Geriatr 2012;12:1-10.

10. Gladman J, Porock D, Griffiths A, et al. Care of older people with cognitive impairment in general hospitals. Final report NIHR Service Delivery and Organisation Programme. 2012. http://www.netscc.ac. uk/hsdr/projdetails.php?ref=08-1809-227 
11. Harwood RH, Porock D, King N, et al. Development of a specialist medical and mental health unit for older people in an acute general hospital. Medical Crises in Older People discussion paper series, issue 5, University of Nottingham. 2010:1-46. http://nottingham.ac. uk/mcop/documents/papers/mcop-issn2044-4230-issue5.pdf

12. Kitwood T. Dementia reconsidered: the person comes first. Buckingham: Open University Press, 1997.

13. Brooker D. Person-centred dementia care. London \& Philadelphia: Jessica Kingsley Publishers, 2007.

14. Pool J. The Pool Activity level (PAL) instrument for occupational profiling: a practical resource for people with cognitive impairments. 3rd edn. London: Jessica King Publishers, 2008.

15. Harwood RH, Goldberg SE, et al. Study protocol. 2011. Evaluation of a Medical and Mental Health Unit compared with standard care for older people whose emergency admission to an acute general hospital is complicated by concurrent 'confusion': a controlled clinical trial. Trials 2011;12:1-11. http://www.trialsjournal.com/content/12/1/ 123

16. Goldberg SE, Bradshaw LE, Kearney FC, et al. Care in a specialist Medical and Mental Health Unit compared with standard care for older people with cognitive impairment admitted to a general hospital: a randomised controlled trial (NIHR TEAM trial). BMJ 2013;347: $\{4132$.

17. Ritchie J, Spencer L. Qualitative data analysis for applied policy research. In: Bryman A, Burgess RG, eds. Analyzing qualitative data. London: Routledge, 1994. 173-194.

18. Srivastava A, Thomson SB. Framework analysis: a qualitative methodology for applied policy research. $J$ Adm Governance 2009;4:72-9. http://www.joaag.com/uploads/06_Research_Note_ Srivastava_and_Thomson_4_2_.pdf

19. Rozenblum R, Lisby M, Hockley PM, et al. Uncovering the blind spot of patient satisfaction: an international survey. BMJ Qual Saf 2011;20:959-65.

20. Brooker D. What is person-centred care in dementia? Rev Clin Gerontol 2003;13:215-22. http://journals.cambridge.org/action/ displayAbstract?fromPage $=$ online\&aid $=230595$

21. Edvardsson D, Winblad B, Sandman PO. Person-centred care of people with severe Alzheimer's disease: current status and ways forward. Lancet Neurol 2008;4:362-7.

22. Clissett $\mathrm{P}$, Porock $\mathrm{D}$, Harwood $\mathrm{RH}$, et al. The challenges of achieving person-centred care in acute hospitals: a qualitative study of people with dementia and their families. Int J Nurs Stud 2013; (11):1495-503. http://dx.doi.org/10.1016/j.jinurstu.2013.03.001

23. Clarke A, Hanson EJ, Ross $\mathrm{H}$. Seeing the person behind the patient: enhancing the care of older people using a biographical approach. J Clin Nurs 2003;12:697-706. http://www.ncbi.nlm.nih.gov/pubmed/ 12919216

24. Day K, Carreon D, Stump C. The therapeutic design of environments for people with dementia. Gerontologist 2000;40:397-416. http://www. ncbi.nlm.nih.gov/pubmed/10961029

25. Lawton MP. The physical environment of the person with Alzheimer's disease. Aging Ment Health 2001;5:56-64. http://www. ncbi.nlm.nih.gov/pubmed/11513499

26. Overshott R, Burns A. Non-pharmacological treatment of severe dementia: an overview. In: Burns A, Winblad B, eds. Server dementia. Chichester: John, Wiley \& Sons Ltd, 2006:164-75.
27. Edvardsson D, Fetherstonhaugh D, Nay R. Promoting a continuation of self and normality: person-centred care as described by people with dementia, their family members and aged care staff. J Clin Nurs 2010;19:2611-18.

28. Whittamore KH, Goldberg SE, Gladman J, et al. The diagnosis, prevalence and outcome of delirium in a cohort of older people with mental health problems on general hospital wards. Int $J$ Geriatr Psychiatry 2014;29:32-40

29. Borbasi S, Jones J, Lockwood C, et al. Health professionals' perspectives of providing care to people with dementia in the acute setting: toward better practice. Geriatr Nurs 2006;27:300-8. http:// www.ncbi.nlm.nih.gov/pubmed/17045129

30. Adams T, Gardiner P. Communication and interaction within dementia care triads. Developing a theory for relationship person-centred care. Dementia 2005;4:185-205.

31. Nolan MR, Davies S, Brown J, et al. Beyond person-centered care: a new vision for gerontological nursing. J Clin Nurs 2004;13:45-53. http://www.ssiacymru.org.uk/resource/9_n_Beyond_Person_ Centred_Care.pdf

32. Tolson D, Smith M, Knight P. An investigation of the components of best nursing practice in the care of acutely ill hospitalized older patients with coincidental dementia: a multi-method design. J Adv Nurs 1999;30:1127-36. http://onlinelibrary.wiley.com/doi/10.1046/j. 1365-2648.1999.01194.x/pdf

33. Bradshaw LE, Goldberg SE, Schneider JM, et al. Carers' for older people with co-morbid cognitive impairment in general hospital: characteristics and psychological well-being. Int $J$ Geriatr Psychiatry 2013;28:681-90.

34. Li H, Powers BA, Melnyk BM, et al. Randomized controlled trial of CARE: an intervention to improve outcomes of hospitalized elders and family caregivers. Res Nurs Health 2012;35:533-49.

35. Waller S. Redesigning wards to support people with dementia in hospital. Nurs Older People. 2012;24:16-21. http://nursingolderpeople. rcnpublishing.co.uk/archive/article-redesigningwards-to-support-people-with-dementia-in-hospital

36. Nufer TW, Spichiger E. How family carers of people with dementia experienced their stay on an acute care facility and their own collaboration with professionals: a qualitative study. Pflege 2011:24:229-37.

37. Lindhardt T, Nyber P, Hallberg IR. Collaboration between relatives of elderly patients and nurses and it's relation to satisfaction with the hospital care trajectory. Scand J Caring Sci 2008;22:507-19.

38. Tadd W, Hillman A, Calnan S, et al. Dignity in practice: an exploration of the care of older adults in acute NHS Trusts. (A research summary). NIHR Service Delivery and Organisation Programme. 2011. http:// www.sdo.nihr.ac.uk/projdetails.php? $\mathrm{ref}=08-1819-218$

39. Care Quality Commission. Dignity and nutrition inspection programme: national overview. 2011. http://www.cqc.org.uk/sites/ default/files/media/documents/20111007_dignity_and nutrition inspection_report_final_update.pdf

40. Royal College Nursing (RCN). Dementia: commitment to the care of people with dementia in hospital settings. London. 2013. http://www. rcn.org.uk/_data/assets/pdf_file/0011/480269/004235.pdf

41. Alzheimer's Society. Dementia 2012: a national challenge. London: Alzheimer's Society, 2012. http://www.alzheimers.org.uk/ dementia2012 